\title{
Role of cysteinyl-leukotrienes for portal pressure regulation and liver damage in cholestatic rat livers
}

\author{
Mark op den Winkel ${ }^{1}$, Leonore Gmelin ${ }^{1}$, Julia Schewe ${ }^{1}$, Natalie Leistner ${ }^{1}$, Manfred Bilzer², Burkhard Göke ${ }^{1}$, \\ Alexander L Gerbes ${ }^{1}$ and Christian J Steib ${ }^{1}$
}

Kupffer cells (KCs) have a major role in liver injury, and cysteinyl-leukotrienes (Cys-LTs) are known to be involved as well. The KC-mediated pathways for the production and secretion of Cys-LT in cholestatic liver injury have not yet been elucidated. Here, we hypothesized that KC activation by Toll-like receptor ligands results in Cys-LT-mediated microcirculatory alterations and liver injury in acute cholestasis. We hypothesized further that this situation is associated with changes in the secretion and production of Cys-LT. One week after bile duct ligation (BDL), livers showed typical histological signs of cholestatic liver injury. Associated microcirculatory disturbances caused increased basal and maximal portal pressure following $\mathrm{KC}$ activation. These differences were determined in BDL livers compared with shamoperated livers in vivo (KC activation by LPS $4 \mathrm{mg} / \mathrm{kg}$ b.w.) and in isolated perfused organs (KC activation by Zymosan A, $150 \mu \mathrm{g} / \mathrm{ml}$ ). Treatment with the 5-lipoxygenase inhibitor MK-886 alone did not alter portal perfusion pressure, lactate dehydrogenase (LDH) efflux, or bile duct proliferation in BDL animals. Following KC activation, portal perfusion pressure increased. The degree of cell injury was attenuated by MK-886 $(3 \mu \mathrm{M})$ treatment as estimated by LDH efflux. In normal rats, a large amount of Cys-LT efflux was found in the bile. Only a minor amount was found in the effluent perfusate. In BDL livers, the KC-mediated Cys-LT efflux into the sinusoidal system increased, although the absolute Cys-LT level was still grossly lower than the biliary excretion in sham-operated livers. In conclusion, our results indicate that treatment with Cys-LT inhibitors might be a relevant target for attenuating cholestatic liver damage.

Laboratory Investigation (2013) 93, 1288-1294; doi:10.1038/labinvest.2013.115; published online 23 September 2013

KEYWORDS: bile duct ligation; cholestatic liver disease; Kupffer cell; microcirculation; MK-886; portal pressure

Leukotrienes are mediators of liver injury in various liver diseases. ${ }^{1-3}$ Accordingly, the inhibition of leukotriene synthesis or the blockade of cysteinyl-leukotriene (Cys-LT) receptor 1 has been shown to protect against liver injury. ${ }^{4,5}$ However, elevated leukotriene synthesis is not detected in all forms of acute liver injury. In addition, it is known that the elimination of Cys-LTs is altered in some pathological conditions. ${ }^{6}$

Different mechanisms of Cys-LT-related liver injury have been identified; the two major postulated mechanisms are intrahepatic vasoconstriction leading to hypoxia ${ }^{7-9}$ and inflammatory cell damage. ${ }^{8,10}$ Furthermore, Kupffer cells (KCs) have an important role in Cys-LT-mediated necroinflammatory liver injury. $^{2}$ The inhibition of 5-lipoxygenase (5-LO)-activating protein (FLAP) resulted in the partial depletion of KCs protecting the liver. ${ }^{2}$ In addition, Cys-LTs are known as mediators of cholestasis. ${ }^{8,11,12}$ They are thought to increase the vascular permeability that prompts hepatic edema. ${ }^{13}$ This results in an increased resistance to bile flow and eventual cholestasis. ${ }^{11}$

To date, the effects of Cys-LT on liver injury and hepatic microcirculation in acute cholestasis are unclear. The present study elaborates microcirculatory alterations and $\mathrm{KC}$-associated pathways of Cys-LT excretion and production resulting in liver injury. The inhibition of 5-LO resulted in reduced portal perfusion pressure and lactate dehydrogenase (LDH) efflux following KC activation in animals 1 week after bile duct ligation (BDL).

\section{MATERIALS AND METHODS Animal Studies}

All animals were treated ethically according to the criteria defined by the National Academy of Sciences and as published by the National Institutes of Health, in addition to

\footnotetext{
${ }^{1}$ Department of Medicine II, Hospital of the Ludwig-Maximilians University (LMU) of Munich, Campus Grosshadern, Munich, Germany and ${ }^{2}$ MSD Sharp \& Dohme $\mathrm{GmbH}$, Haar, Germany

Correspondence: Dr CJ Steib, MD, Department of Medicine II, Hospital of the Ludwig-Maximilians University (LMU) of Munich, Grosshadern, Marchioninistraße 15, 81377 Munich, Germany.

E-mail: christian.steib@med.uni-muenchen.de

Received 8 December 2012; revised 6 August 2013; accepted 28 August 2013
} 
the legal requirements in Germany. All animal studies were approved by the local government (Regierung von Oberbayern, Munich, Germany) and were reported to the responsible authorities regularly.

\section{Induction of Cholestasis by $B D L$}

Male Sprague-Dawley rats were anesthetized by intraperitoneal pentobarbital injection ( $50 \mathrm{mg} / \mathrm{kg}$ body weight). After a midline laparotomy was performed, the common bile duct was ligated twice with 3-0 silk and cut between the two ligations. Liver perfusion with Krebs Henseleit buffer or in vivo portal pressure measurements were performed 1 week (BDL) after the operation, as described below. Sham-operated animals were operated accordingly but without ligation and cutting of the bile duct.

\section{In Vivo Measurement of Portal Pressure}

Body weight was determined directly before performing all experiments. Surgery (1 week after BDL or sham operation) commenced after the intraperitoneal injection of sodium pentobarbital $(50 \mathrm{mg} / \mathrm{kg}$ b.w.). Arterial blood pressure was continuously monitored via a carotid catheter. A laparotomy was performed, and a PE-tube $(23 \mathrm{G}, 0.6 \times 30 \mathrm{~mm})$ was inserted over the ileocolic vein and advanced toward the confluence of the portal and splenic veins. ${ }^{9,14,15}$ This cannula was used for the simultaneous infusion of LPS (LPS from E. coli $026: \mathrm{B} 6,4 \mathrm{mg} / \mathrm{kg}$ body weight, $100 \mu \mathrm{l} / \mathrm{min}$ ) and to monitor portal pressure in sham-operated and BDL rats (each $n=6$ ) via a transducer system (Sirecust 404 from Siemens, Germany, and Transducer Gabarith from Becton Dickinson, Singapore) as described elsewhere. ${ }^{14}$

\section{In Situ Rat Liver Perfusion Study}

Sham-operated male Sprague-Dawley rats (Charles River, Sulzfeld, Germany) or male Sprague-Dawley rats 1 week after BDL (see section above) were anesthetized with an intraperitoneal injection of sodium pentobarbital $(50 \mathrm{mg} / \mathrm{kg}$ body weight). Afterwards, the portal vein was cannulated with a 14-G Teflon intravenous catheter, and the liver was perfused at a constant flow rate. The inferior vena cava was cannulated via the right atrium and ligated above the right renal vein. In all of the experiments, the livers were perfused with KrebsHenseleit solution $\left(\mathrm{pH} 7.4,37^{\circ} \mathrm{C}\right)$ in a non-recirculating manner. The perfusion buffer was gassed with a mixture of $95 \% \mathrm{O}_{2}$ and $5 \% \quad \mathrm{CO}_{2}$ using an oxygenator. ${ }^{5}$ The portal perfusion pressure was monitored continuously. The bile duct was cannulated with polyethylene tubing (PE 10) to monitor the bile flow in the non-operated animals. Cannulating the bile duct was not possible in BDL animals. The liver was allowed to stabilize for $20 \mathrm{~min}$ before any substances were added. During this stabilization period, the criteria for liver viability included the following: stable perfusion pressure and bile production $>0.8 \mu \mathrm{l} / \mathrm{min} \times \mathrm{g}$ liver (in animals that were sham-operated). LDH-activity was analyzed at several time points in each animal (minutes $20,30,40,44,46,50,60,80$ ) according to standard tests. ${ }^{9,14}$ If one of the viability criteria was not met, the experiment was terminated.

\section{Activation of KC}

KCs were activated by the infusion of cell wall particles from Saccharomyces cerevisiae (Zymosan A). Zymosan suspensions prepared as earlier described $(150 \mu \mathrm{g} / \mathrm{ml})^{14,15}$ were infused from 40 to $46 \mathrm{~min}$ after the start of liver perfusion. In a first set of experiments, portal perfusion pressure, LDH-efflux, and bile flow from sham-operated rat livers perfused with Krebs-Henseleit buffer only $(n=6)$ were compared with the same parameters in Zymosan-treated livers $(n=6)$. Consecutive experiments compared the portal perfusion pressure and LDH-efflux of Zymosan-treated animals 1 week after BDL $(n=6)$ with livers $(n=6)$ by Zymosan.

\section{Inhibition of 5-LO Activation Through MK-886}

The effects of leukotrienes were investigated through the inhibition of 5-LO activation using MK-886. In the first set of experiments, MK-886 alone was infused from 20 to $50 \mathrm{~min}$ (final concentration $3 \mu \mathrm{M}, n=6)$. In the second set of experiments, Zymosan (from 40 to $46 \mathrm{~min}$ ) was infused together with MK-866 (final concentration $3 \mu \mathrm{M}$ ) in DMSO (from 20 to $50 \mathrm{~min}$ ) in sham-operated and bile duct-ligated livers (each $n=6$ ).

\section{ELISA}

The release of Cys-LTs ( $\mathrm{LT} \mathrm{C}_{4}, \mathrm{D}_{4}$, and $\mathrm{E}_{4}$ ) into the effluent perfusate and bile was quantified using an enzyme immunoassay (Cayman Chemical, Ann Arbor, MI, USA). The Cys-LT content of sham-operated livers compared with bile duct-ligated livers was measured using the same enzyme immunoassay.

\section{Histological Evaluation}

Liver tissue was fixed in $4 \%$ buffered formalin, dehydrated in graded ethanol, and embedded in paraffin using standard methods. Then $4-\mu \mathrm{m}$ longitudinal sections were stained with $\mathrm{H} \& \mathrm{E}$ (hematoxylin/eosin) and Elastica van Gieson (EvG) according to standard procedures. Scoring systems were used to compare inflammation, bile duct proliferation, fibrosis, and necrosis of the different histological pictures.

\section{Drugs and Reagents}

LPS, MK-886, and Zymosan were obtained from Sigma Chemical CO (St Louis, MO, USA). MK-886 was prepared as a DMSO stock solution. The stock solution was infused into the portal inflow of the perfusion system by micro-infusion pumps at a rate of $100 \mu \mathrm{l} / \mathrm{min}$. Zymosan was dissolved directly in perfusion buffer.

\section{Statistical Analysis}

All data are expressed as the mean \pm standard deviation or standard error of the mean. Statistical analyses of the data were performed using the Mann-Whitney $U$-test for nonnormally distributed data or Student's $t$-test. A value of $P<0.05$ was considered to be statistically significant. 
a

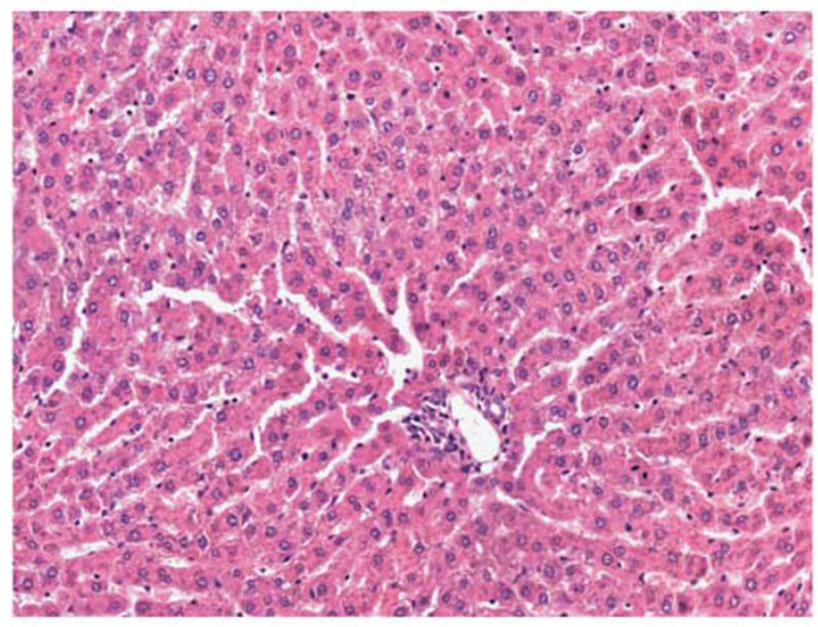

b

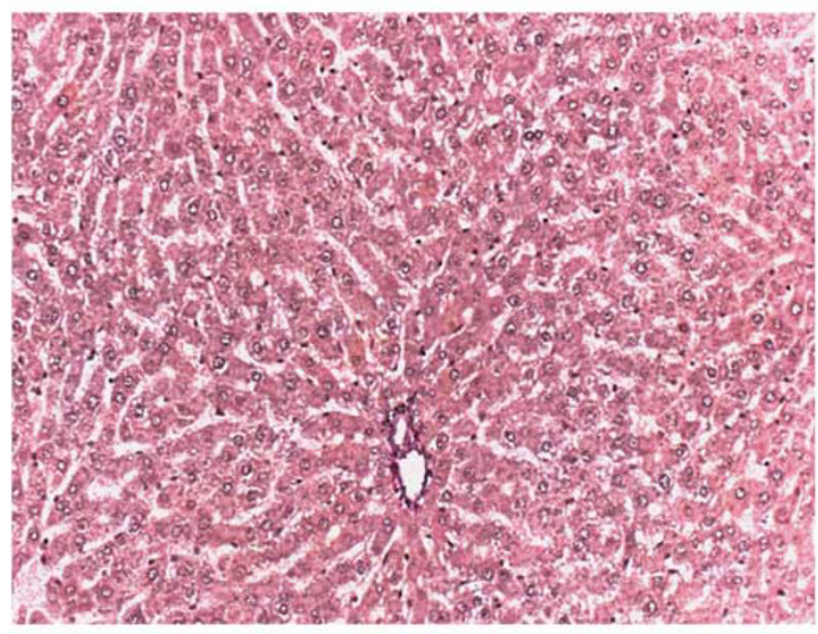

sham

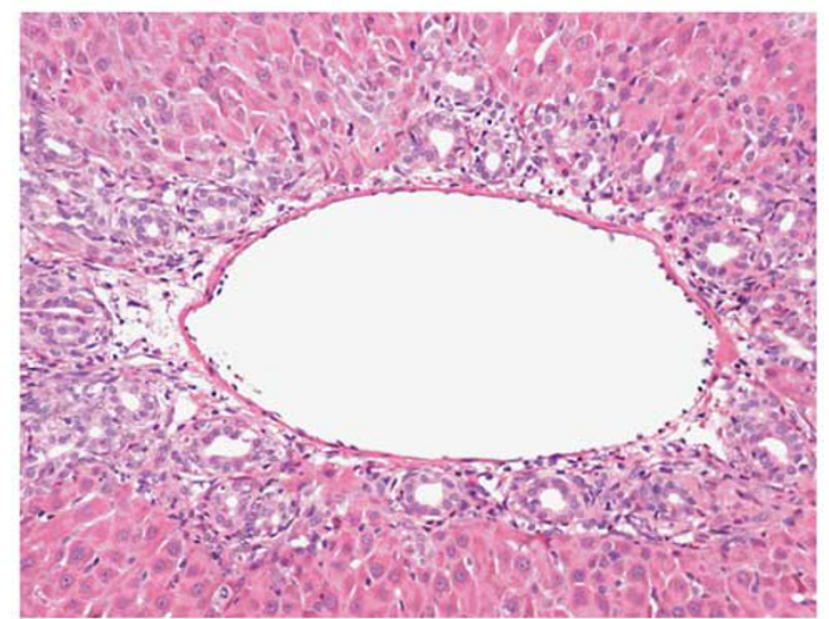

BDL

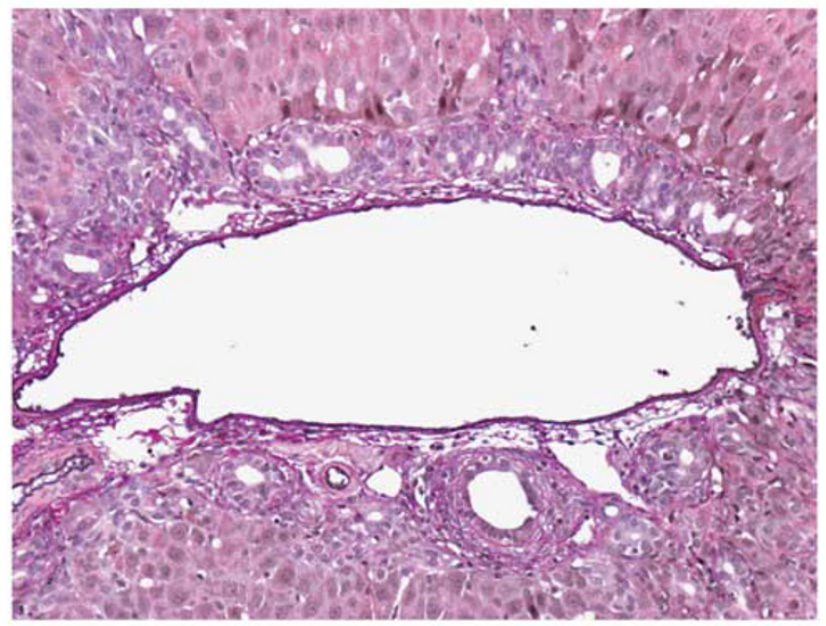

BDL

Figure 1 Histological evaluation of livers with acute cholestasis. (a) Hematoxylin/eosin (H\&E) staining of the livers 1 week after bile duct ligation showed typical signs of liver injury and cholestasis (amplified portal fields with a mixed infiltration in addition to bile duct proliferation). Sham-operated animals showed normal liver architecture. (b) Elastica van Gieson (EvG) staining showed the beginning of fibrotic alterations in livers 1 week after bile duct ligation; Sham-operated livers showed no signs of cholestasis.

\section{RESULTS}

\section{Histological Evaluation}

Cholestasis induced by BDL for 1 week resulted in typical histological signs of liver injury and cholestasis. The portal fields of the livers were broadened and showed mixed infiltration in animals that had been bile duct-ligated for 1 week; bile duct proliferation and very mild fibrosis were seen in these livers as well (Figures 1a and b). Sham-operated livers showed typical liver architecture (Figures $1 \mathrm{a}$ and $\mathrm{b}$ ).

\section{Enhancement of the Increase in Portal Pressure after BDL}

Portal pressure measurements in vivo showed that the minimal and the maximal portal pressure increased following infusion of the Toll-like receptor (TLR) 4 ligand LPS via the portal vein 1 week after BDL compared with sham-operated livers (Figure 2a). The absolute increase in portal pressure 1 week after BDL was also enhanced compared with that observed in sham-operated livers. In isolated perfused organs, the portal perfusion pressure was analyzed at the onset of the experiments. Here, the basal portal perfusion pressure was lower in normal livers than in livers after BDL (Figure 2b). After KC activation by Zymosan A, the portal perfusion pressure increased to a higher maximum 1 week after BDL compared with sham-operated livers (Figure 2b). At the end of the experiments, the portal perfusion pressure returned to about baseline levels in sham-operated livers but remained elevated in BDL livers (Figure 2b). Even the absolute increase in portal perfusion pressure increased 1 week after BDL compared with controls. 
a
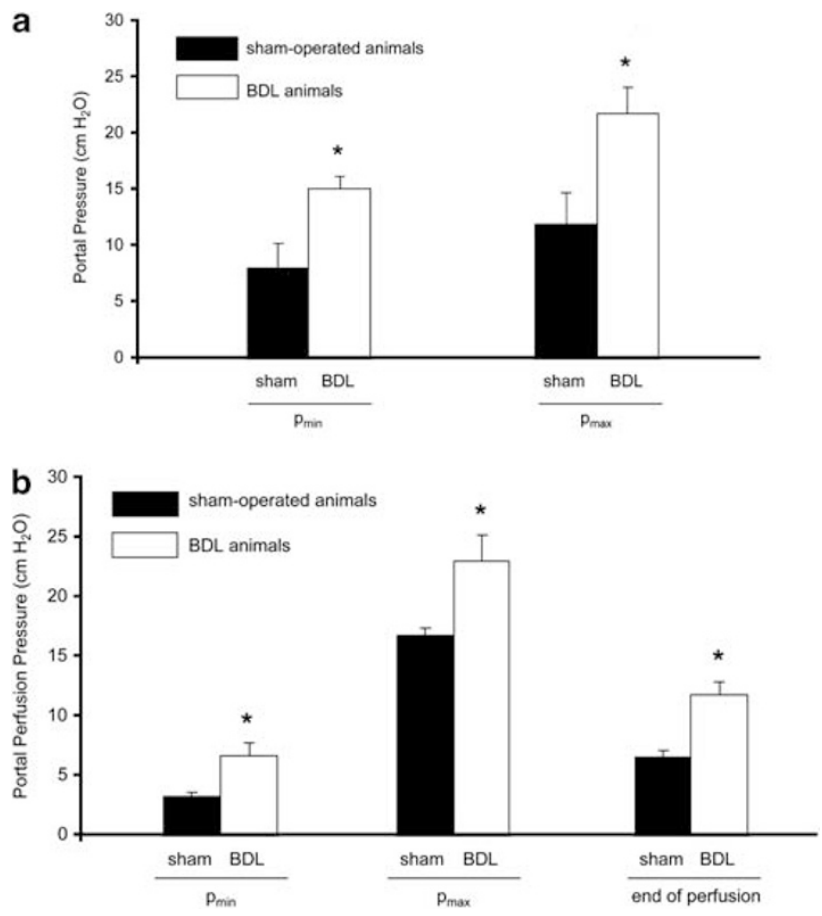

Figure 2 Increase in portal pressure following Kupffer cell (KC) activation in acute cholestasis. Data are expressed as the mean \pm s.d. (a) The determination of portal pressure in vivo revealed a significant $\left({ }^{*} P<0.05\right)$ increase in basal portal pressure $\left(p_{\mathrm{min}}\right)$ and in maximal portal pressure $\left(p_{\max }\right)$ following KC activation by LPS (LPS from E. coli 026:B6, $4 \mathrm{mg} / \mathrm{kg}$ body weight, $100 \mu \mathrm{l} / \mathrm{min}$ ) in cholestatic livers 1 week after bile duct ligation $(n=6)$ compared with sham-operated livers $(n=6)$. (b) Basal portal perfusion pressure in situ was significantly $\left({ }^{*} P<0.05\right)$ higher in animals 1 week after bile duct ligation (BDL) than in sham-operated animals (each $n=6$ ). Maximal portal perfusion pressure following the infusion of Zymosan $(150 \mu \mathrm{g} / \mathrm{ml})$ from 40 to $46 \mathrm{~min}$ after starting liver perfusion was also significantly $(* P<0.05)$ higher in $B D L$ livers than in sham-operated livers (each $n=6$ ). Portal perfusion pressure at the end of perfusion remained significantly elevated in BDL compared with normal livers (each $n=6$ ).

\section{Cys-LT-Related Liver Injury}

The infusion of MK-886 alone to inhibit 5-LO activation in livers 1 week after BDL did not influence portal perfusion pressure compared with perfusion experiments without any treatment (Figure 3a). Accordingly, LDH efflux to estimate liver injury was not changed by infusion of MK-886 (Figure $3 \mathrm{~b}$ ) and the histological picture was not altered by MK-886 treatment (Figure 3c). Interestingly, bile duct proliferation estimated via a scoring system by EvG staining was also not influenced by MK- 886 treatment. Infusion of MK886 in sham-operated livers and livers examined 1 week after $\mathrm{BDL}$ in addition to $\mathrm{KC}$ activation by Zymosan reduced maximal portal perfusion pressure in both experimental settings (Figure 4a). Measurements of LDH in the effluent perfusate were used to estimate the extent of liver injury. Maximal LDH efflux in normal livers was only slightly reduced by the additional treatment with MK-866 compared with $\mathrm{KC}$ activation by Zymosan alone (Figure $4 \mathrm{~b}$ ). The maximal LDH efflux was significantly $(P<0.05)$ higher in cholestatic livers compared with sham-operated livers. The LDH efflux was reduced by additional treatment with MK-886 in BDL animals (Figure 3d).

\section{Mechanism of Cys-LT Secretion and Production}

In the next step, we investigated Cys-LT production in the effluent perfusate. We postulated an increase in Cys-LT production correlating to the increase in portal perfusion pressure and LDH efflux. In sham-operated livers, the sinusoidal Cys-LT efflux into the effluent perfusate increased with $\mathrm{KC}$ activation, but not significantly (Figure 5a). Higher amounts of Cys-LT were found in the bile following KC activation compared with the sinusoidal efflux of Cys-LT in sham-operated animals (Figure 5b). The bile flow over 80 min was stable in the group treated with DMSO alone and the group treated with MK-886 (data not shown). When the bile duct was ligated, biliary Cys-LT efflux was no longer possible. Following BDL, the magnitude of sinusoidal Cys-LT efflux increased following $\mathrm{KC}$ activation compared with that observed at the start of perfusion experiments (Figure 5a). However, the absolute efflux was still low compared with the biliary efflux in sham-operated livers.

\section{DISCUSSION}

This study investigated the mechanisms of liver injury following KC activation in rat livers that had been bile ductligated for 1 week in comparison to sham-operated livers. There were three principal findings in our study. First, in cholestatic livers 1 week after BDL, both the basal and the maximal portal pressure following $\mathrm{KC}$ activation were impressively higher as compared with sham-operated livers. Second, increased portal perfusion pressure is associated with more intense liver damage, which could be abolished by the inhibition of 5-LO activation through the use of MK-886. Finally, Cys-LTs were secreted into the bile to a much higher degree than to the sinusoidal system in sham-operated livers. When the bile duct was obstructed in cholestatic livers, the Cys-LT efflux into the sinusoidal system was still low compared with the biliary Cys-LT efflux observed in sham-operated livers.

These experiments were performed in vivo and in the system of isolated organs in situ. The first set of experiments in the present study showed that the portal pressure was enhanced in cholestatic livers 1 week after BDL compared with sham-operated livers. This was in accordance with the findings of earlier studies. ${ }^{16}$ Microcirculatory disturbances and liver injury have been shown to be interrelated in animal studies and human studies in the setting of ischemia reperfusion, endotoxin-induced liver injury, or druginduced liver injury. ${ }^{17-20}$ Accordingly, in the current study, LDH efflux from isolated perfused livers increased to a higher degree in cholestatic livers than in sham-operated livers following KC activation. The good correlation between $\mathrm{LDH}$ efflux and transaminases has been shown in different earlier studies. ${ }^{21,22}$ 


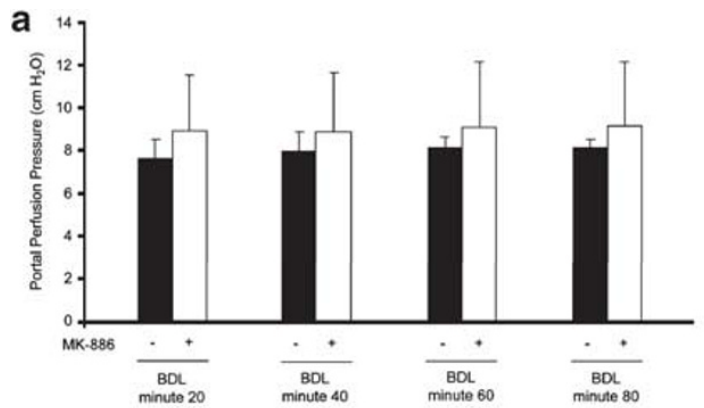

C

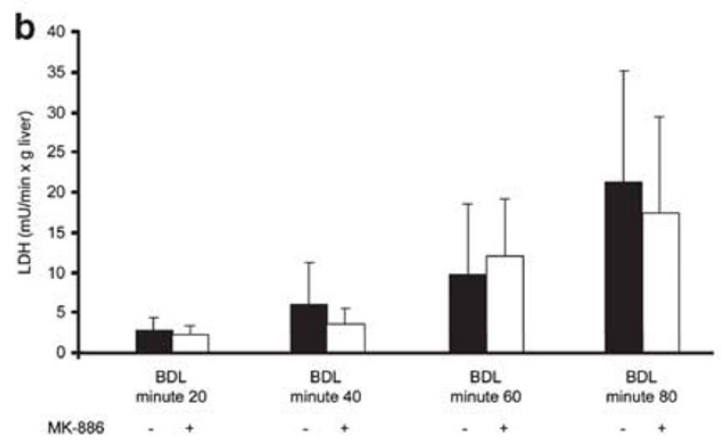

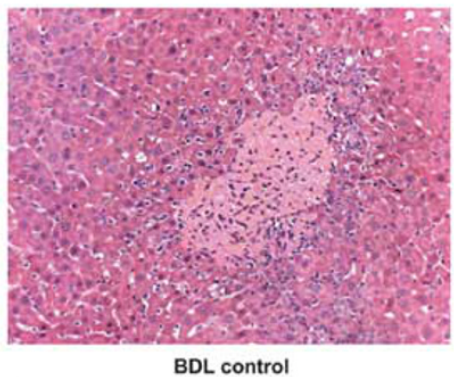

d

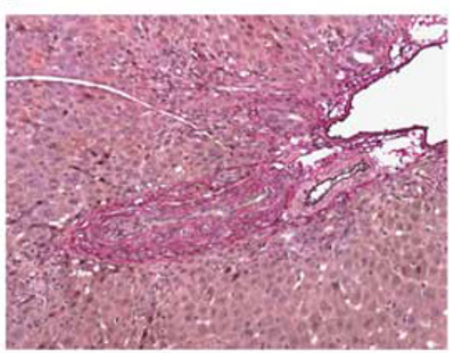

BDL control

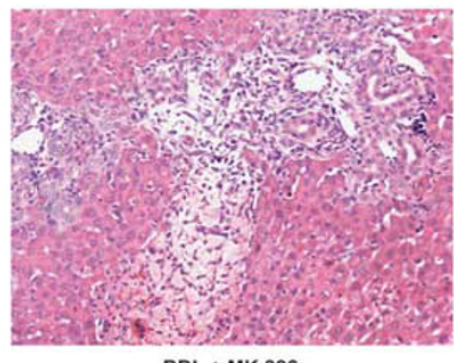

$\mathrm{BDL}+\mathrm{MK} 886$

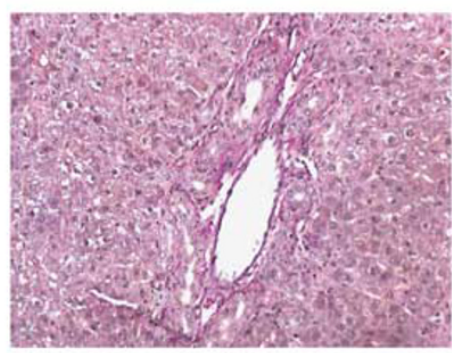

$B D L+M K 886$

Figure 3 Effect of 5-lipoxygenase (5-LO) inhibition by MK-886 on portal perfusion pressure and liver injury. Data are expressed as the mean \pm s.d. (a) The inhibition of 5-LO activation by the infusion of MK-886 $(3 \mu \mathrm{M}, n=6)$ from 20 to $50 \mathrm{~min}$ did not alter portal perfusion pressure in BDL animals. (b) Accordingly, LDH efflux as a sign of cell damage was not influenced by MK-886 treatment $(3 \mu \mathrm{M}, n=6)$ when infused from 20 to 50 min. (c) H\&E staining showed no changes concerning liver injury following MK-886 treatment from 20 to 50 min compared with control animals. (d) Elastica van Gieson staining to estimate bile duct proliferation showed no influence on bile duct proliferates in MK-886-treated animals compared with untreated controls.

Previous studies provide solid evidence that Cys-LTs have an important role in liver injury. Leukotrienes are involved in different settings of acute liver injury, for example, in hepatic ischemia reperfusion injury, ${ }^{23}$ or in the model of endotoxin and D-galactosamine administration leading to liver injury. ${ }^{5}$ One postulated mechanism is the activation of chloride conductance in hepatocytes. ${ }^{3}$

An interesting new aspect of Cys-LT-induced liver injury in cholestasis is described here. After BDL, Cys-LTs can no longer be excreted via the bile. Following KC activation, Cys-LTs increased in the sinusoidal systems of cholestatic livers. However, inhibition of 5-LO by MK-886 alone did not influence portal perfusion pressure, LDH efflux, or histological alterations. Lower amounts of Cys-LTs were found in the sinusoidal system following KC activation in sham-operated livers, but extremely large amounts of Cys-LTs were excreted via the bile. Accordingly, the inhibition of 5-LO activation almost completely abolished Cys-LT excretion into the bile in shamoperated livers. Following KC activation, LDH efflux was higher in cholestatic livers than in sham-operated animals; LDH efflux was reduced in BDL animals through the inhibition of 5-LO activation, but only to a minor degree in shamoperated animals. Hence, Cys-LTs produced after KC activation are more relevant mediators of liver injury in cholestasis, when secretion of Cys-LTs via bile is not any more possible.

This finding is of clinical relevance for cholestatic liver diseases such as primary biliary cirrhosis or primary sclerosing cholangitis. It is of high interest for the pathophysiology of obstruction of the bile duct by gallstones, sclerosis or stenosis of the bile duct, ischemic-type biliary lesions, or biliary casts following liver transplantation. In the setting of KC activation (for example, by TLR ligands like bacterial products), this finding becomes even more relevant as the clinical settings of bile duct obstruction are often associated with the presence of bacterial products.

Following BDL, not only the number of KC increases ${ }^{24}$ but also the number of cholangiocytes and bile duct proliferates. ${ }^{25,26}$ On the other hand, there are no data about Cys-LT production and 5-LO expression in cholangiocytes and bile duct proliferates up to now. Interestingly, MK-886 treatment did not influence bile duct proliferates. Accordingly, the general opinion about production of Cys-LT is that Leukotriene A is produced in $\mathrm{KC}$ via $5-\mathrm{LO}$ and Cys-LT are produced from Leukotriene A via Leukotriene C4 synthase mainly found in hepatocytes. ${ }^{6}$

The increased Zymosan-induced sinusoidal Cys-LT release could explain the augmented Zymosan-induced portal perfusion pressure increase in BDL animals. Large amounts of Cys-LTs are secreted via bile in sham-operated animals. The obstruction of the bile duct leads to enhanced efflux of CysLTs into the sinusoidal perfusate. In cholestatic livers, therefore, treatment with MK-886 becomes more effective in view of liver injury. Concerning attenuation of portal perfusion pressure, MK-886 treatment has significant effects in 


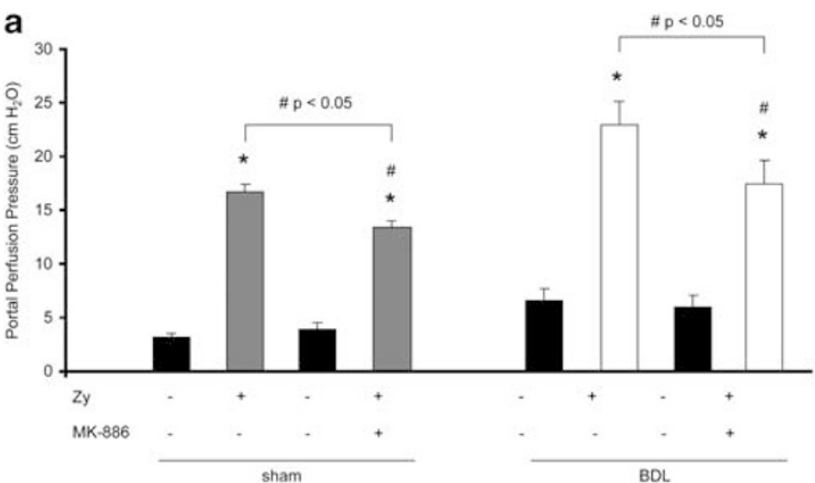

b

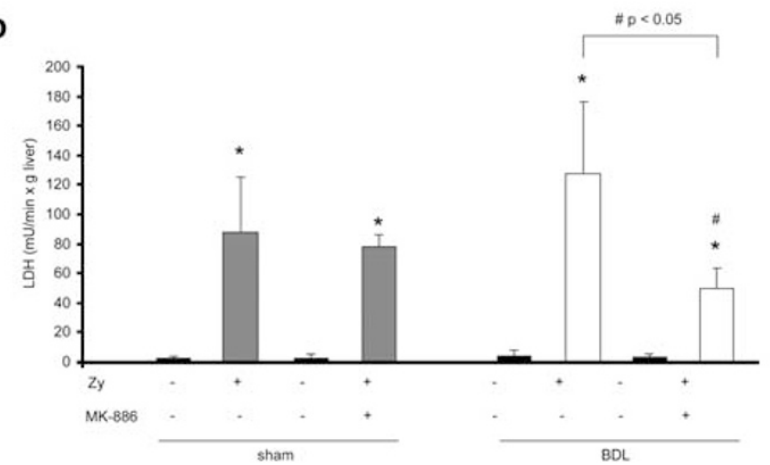

Figure 4 Effect of 5-lipoxygenase (5-LO) inhibition by MK-886 on portal perfusion pressure and liver injury following KC activation. Data are expressed as the mean \pm s.d. (a) Activation of KC by Zymosan infusion (Zy, $150 \mu \mathrm{g} / \mathrm{ml}$, from 40 to $46 \mathrm{~min}$ after starting liver perfusion) of shamoperated livers and of cholestatic livers (each $n=6$ ) in addition to treatment with the solvent DMSO (20-50 min) was accompanied by a marked increase in portal perfusion pressure $\left({ }^{*} P<0.05\right)$. The inhibition of 5-LO activation by the infusion of MK-886 ( $3 \mu \mathrm{M}, n=6)$ from 20 to $50 \mathrm{~min}$ significantly $(\# P<0.05)$ reduced this maximal increase in portal perfusion pressure after KC activation by Zymosan $(150 \mu \mathrm{g} / \mathrm{ml})$ in both settings $(n=6)$. (b) In sham-operated livers, the infusion of Zymosan $(150 \mu \mathrm{g} / \mathrm{ml})$ from 40 to $46 \mathrm{~min}$ after starting liver perfusion in addition to treatment with the solvent DMSO (2050 min) increased LDH efflux as a sign of cell damage. One week after bile duct ligation, the infusion of Zymosan $(150 \mu \mathrm{g} / \mathrm{ml})$ from 40 to $46 \mathrm{~min}$ after starting liver perfusion in addition to treatment with the solvent DMSO enhanced the increase in LDH efflux as well $(n=6)\left({ }^{*} P<0.05\right)$. This LDH efflux was significantly attenuated by MK-886 treatment ( $\left.3 \mu \mathrm{M}, 20-50 \mathrm{~min}, n=6,{ }^{\#} P<0.05\right)$.

sham-operated animals and in BDL animals. Possibly sinusoidal content of Cys-LTs is more important in view of portal perfusion pressure, but for liver injury, potentially sinusoidal and biliary content have a role. On the other hand, additional vassconstrictors like thromboxane $\mathrm{A}_{2}$ are involved in the intrahepatic contraction in sham-operated animals. ${ }^{16,24}$

However, it cannot be fully excluded that the effect of MK-886 is partly due to unspecific anti-inflammatory activity. Arachidonic acid is stereoselectively oxygenated at carbon 5 by the enzyme 5 -LO. The intervention of a second protein, 5-LO-activating protein (FLAP), is required before 5-LO can become catalytically active. ${ }^{27}$ MK- 886 binds to FLAP with high affinity and prevents 5-LO activation. However, further antiinflammatory activity of MK-886 cannot be excluded. ${ }^{28}$ In
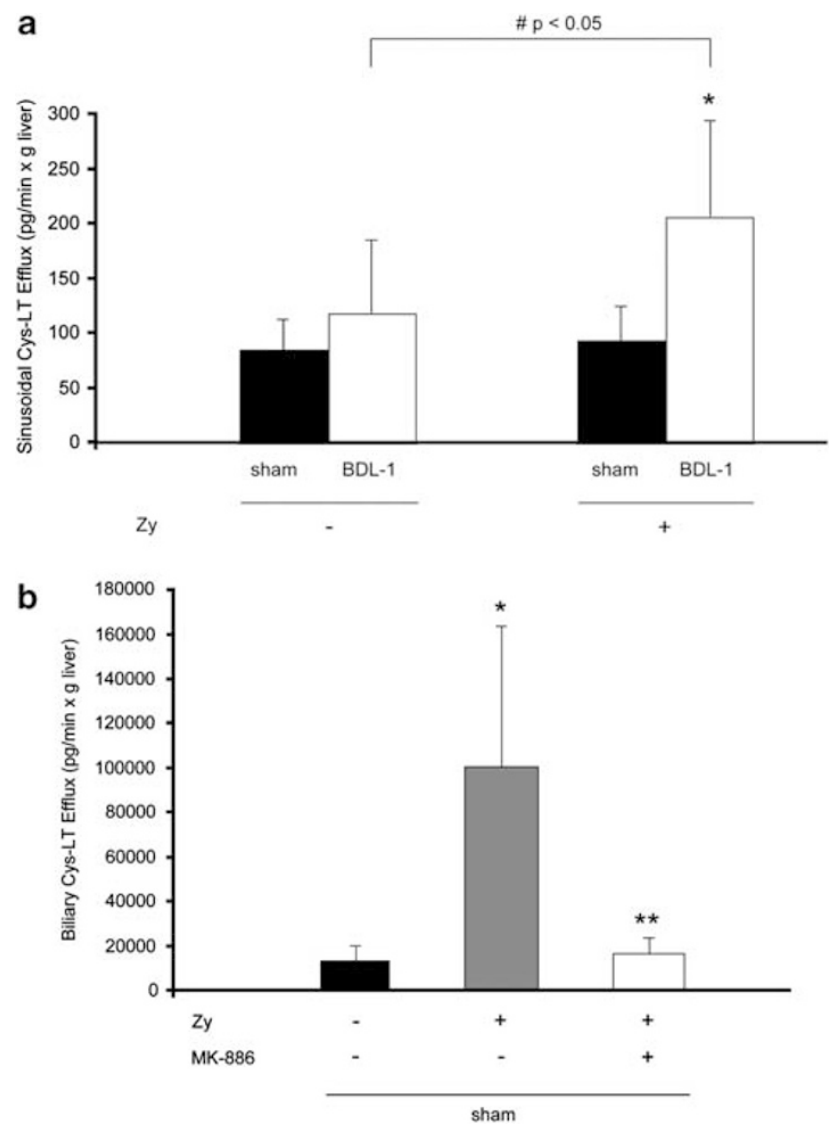

Figure 5 Mechanism of cysteinyl leukotriene (Cys-LT) secretion in acute cholestasis and normal liver. Data are expressed as the mean \pm s.e.m. (a) The infusion of Zymosan ( $Z \mathrm{y}, 150 \mu \mathrm{g} / \mathrm{ml}, n=6)$ from 40 to $46 \mathrm{~min}$ after starting liver perfusion increased the sinusoidal Cys-LT efflux $(\mathrm{pg} / \mathrm{min} \times \mathrm{g}$ liver) compared with the control group that did not undergo Zymosan infusion ( $\left.{ }^{\#} P<0.05\right)$ in BDL animals. Additionally maximal Cys-LT efflux was significantly $\left({ }^{*} P<0.05\right)$ higher in $B D L$ animals than in shamoperated animals. (b) In sham-operated animals, the biliary Cys-LT efflux significantly $\left({ }^{*} P<0.05\right)$ increased after KC activation with Zymosan (150 $\mu \mathrm{g} / \mathrm{ml}, 40-46 \mathrm{~min}, n=6)$ compared with the controls. 5-LO inhibition with MK-886 ( $3 \mu \mathrm{M}, 30-60 \mathrm{~min}, n=6)$ almost completely abolished biliary Cys-LT efflux following KC activation $\left({ }^{* *} P<0.05\right)$.

addition, investigations with MK-886 treatment over a longer time period has been performed in earlier studies ${ }^{29}$ and reduction of fibrosis development has been shown.

In conclusion, Cys-LTs were excreted predominantly via bile in sham-operated livers. Under bile duct obstruction, Cys-LTs are no longer secreted via bile. An additional event such as KC activation, eg, via TLR ligands by bacterial or fungal products, results in Cys-LT-mediated liver injury in cholestatic livers. This liver injury can be abolished by treatment with MK-886, an inhibitor of 5-LO activation. Therefore, inhibitors of 5-LO may offer therapeutic options in clinical situations involving bile duct obstruction.

\section{ACKNOWLEDGMENTS}

We thank I Liss, A Schlossbauer, and Chr v Hesler for excellent technical assistance. We thank Dr Doris Mayr and Dr Marie-Christine Makeschin from 
the Institute of Pathology (LMU, Munich) for the analysis of the histological data. This study is part of Lenore Gmelin's M.D. thesis. This study was supported by grants from the Deutsche Forschungsgemeinschaft (DFG STE 1022/2-1 and STE 1022/2-3) and the faculty of medicine, University of Munich (FöFoLe Reg.-Nr. 396).

\section{DISCLOSURE/CONFLICT OF INTEREST}

The authors declare no conflict of interest.

1. Li G, Chen W, Han C, et al. Cytosolic phospholipase A(2)alpha protects against Fas- but not LPS-induced liver injury. J Hepatol 2011;55:1281-1290.

2. Titos E, Claria J, Planaguma A, et al. Inhibition of 5-lipoxygenaseactivating protein abrogates experimental liver injury: role of Kupffer cells. J Leukoc Biol 2005;78:871-878.

3. Meng XJ, Carruth MW, Weinman SA. Leukotriene D4 activates a chloride conductance in hepatocytes from lipopolysaccharide-treated rats. J Clin Invest 1997;99:2915-2922.

4. Kawada N, Ueda N, Mizoguchi $\mathrm{Y}$, et al. Increased 5-lipoxygenase activity in massive hepatic cell necrosis in the rat correlates with neutrophil infiltration. Hepatology 1992;16:462-468.

5. Tiegs G, Wendel A. Leukotriene-mediated liver injury. Biochem Pharmacol 1988;37:2569-2573.

6. Farzaneh-Far R, Moore K. Cysteinyl-leukotrienes and the liver. Prostaglandins Other Lipid Mediat 2003;72:35-50.

7. Krell $\mathrm{H}$, Dietze E. Hemodynamic and metabolic responses to leukotriene C4 in isolated perfused rat liver. Hepatology 1989;10:300-305.

8. Bilzer $M$, Lauterburg BH. Peptidoleukotrienes increase the efflux of glutathione from perfused rat liver. Prostaglandins Leukot Essent Fatty Acids 1993;49:715-721.

9. Steib CJ, Bilzer $M$, op den Winkel $M$, et al. Treatment with the leukotriene inhibitor montelukast for 10 days attenuates portal hypertension in rat liver cirrhosis. Hepatology 2010;51:2086-2096.

10. Cincu RN, Rodriguez-Ortigosa CM, Vesperinas I, et al. S-adenosyl-Lmethionine protects the liver against the cholestatic, cytotoxic, and vasoactive effects of leukotriene D4: a study with isolated and perfused rat liver. Hepatology 1997;26:330-335.

11. Keppler D, Hagmann W, Rapp S, et al. The relation of leukotrienes to liver injury. Hepatology 1985;5:883-891.

12. Keppler D, Huber M, Baumert T. Leukotrienes as mediators in diseases of the liver. Semin Liver Dis 1988;8:357-366.

13. Hua XY, Dahlen SE, Lundberg JM, et al. Leukotrienes C4, D4 and E4 cause widespread and extensive plasma extravasation in the guinea pig. Naunyn Schmiedebergs Arch Pharmacol 1985;330:136-141.

14. Steib CJ, Hartmann AC, v Hesler C, et al. Intraperitoneal LPS amplifies portal hypertension in rat liver fibrosis. Lab Invest 2010;90:1024-1032.
15. Steib $\mathrm{CJ}$, Hennenberg $M$, Beitinger $F$, et al. Amiloride reduces portal hypertension in rat liver cirrhosis. Gut 2010;59:827-836.

16. Miller AM, Masrorpour M, Klaus C, et al. LPS exacerbates endothelin-1 induced activation of cytosolic phospholipase A2 and thromboxane A2 production from Kupffer cells of the prefibrotic rat liver. J Hepatol 2007;46:276-285.

17. Kelly DM, Shiba H, Nakagawa $S$, et al. Hepatic blood flow plays an important role in ischemia-reperfusion injury. Liver Transpl 2011;17: 1448-1456.

18. Eipel C, Bordel R, Nickels RM, et al. Impact of leukocytes and platelets in mediating hepatocyte apoptosis in a rat model of systemic endotoxemia. Am J Physiol Gastrointest Liver Physiol 2004;286: G769-G776.

19. Li X, Klintman $D$, Liu $Q$, et al. Critical role of CXC chemokines in endotoxemic liver injury in mice. J Leukoc Biol 2004;75:443-452.

20. Ito Y, Abril ER, Bethea NW, et al. Role of nitric oxide in hepatic microvascular injury elicited by acetaminophen in mice. Am J Physiol Gastrointest Liver Physiol 2004;286:G60-G67.

21. Gonzalez-Martin G, Dominguez AR, Guevara A. Pharmacokinetics and hepatotoxicity of diclofenac using an isolated perfused rat liver. Biomed Pharmacother 1997;51:170-175.

22. Toth B, Yokoyama Y, Schwacha MG, et al. Insights into the role of interleukin- 6 in the induction of hepatic injury after traumahemorrhagic shock. J Appl Physiol 2004;97:2184-2189.

23. Yang SL, Huang $X$, Chen HF, et al. Increased leukotriene c4 synthesis accompanied enhanced leukotriene c4 synthase expression and activities of ischemia-reperfusion-injured liver in rats. J Surg Res 2007;140:36-44.

24. Steib CJ, Gerbes AL, Bystron M, et al. Kupffer cell activation in normal and fibrotic livers increases portal pressure via thromboxane $A(2)$. J Hepatol 2007;47:228-238.

25. Omenetti A, Yang L, Gainetdinov RR, et al. Paracrine modulation of cholangiocyte serotonin synthesis orchestrates biliary remodeling in adults. Am J Physiol Gastrointest Liver Physiol 2011;300:G303-G315.

26. Glaser S, Lam IP, Franchitto A, et al. Knockout of secretin receptor reduces large cholangiocyte hyperplasia in mice with extrahepatic cholestasis induced by bile duct ligation. Hepatology 2010;52: 204-214.

27. Abramovitz $M$, Wong $E$, Cox ME, et al. 5-Lipoxygenase-activating protein stimulates the utilization of arachidonic acid by 5 -lipoxygenase. Eur J Biochem 1993;215:105-111.

28. Batt DG. 5-Lipoxygenase inhibitors and their anti-inflammatory activities. Prog Med Chem 1992;29:1-63.

29. El-Swefy S, Hassanen SI. Improvement of hepatic fibrosis by leukotriene inhibition in cholestatic rats. Ann Hepatol 2009;8: 41-49. 